NASA Technical Memorandum 82943

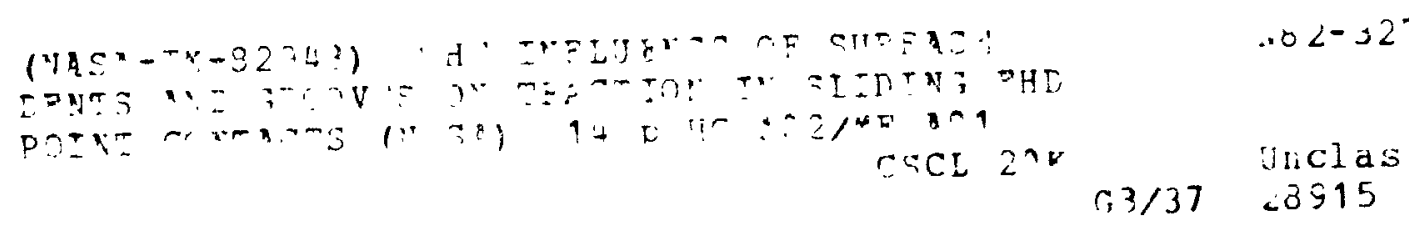

\title{
The Influence of Surface Dents and Groovis on raction in Sliding EHD Point Contacts
}

\section{Cusano}

University of Illinois at Urbana-Champaign

Urbaria, Illinuis

and

L. D. Wedeven

Lewis Research Center

i ieveland, Ohio

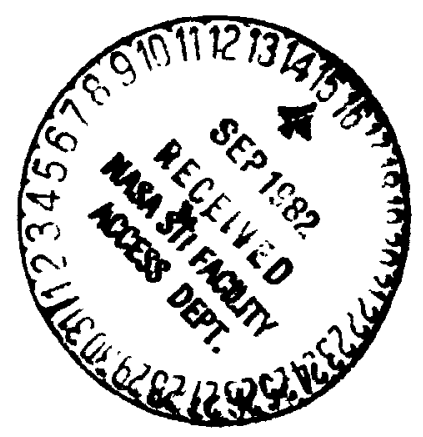

Prepared for the

Joint Lubrication Conference

cosponsored by the American Society of Mechanical Engineers

and the American Society of Lubrication Engineers

Washington, D.C., October 5-7, 1982 


\title{
THE INFLUENCE OF SURFACE DENTS AND GROOVES ON
}

\section{TRACTION IN SLIOING EHD POINT CONTACTS}

\author{
C. Cusano \\ Department of Mechanical and Industrial Engineering \\ University of Illinois at Urbana-Champaign \\ Urbana, IL 61801 \\ and \\ L. D. Wedeven \\ National Aeronautics and Space Administration \\ Lewis Research Center \\ Clevelaind, $\mathrm{OH} 44135$
}

\begin{abstract}
SUMMARY
Changes in traction, caused by dents and grooves on a highly polished ball, are investigated as these defects approach and go through sliding elastohydrodynamic (EHD) point contacts. The contacts are formed with the ball loaded against a transparent disk. The ball and thus the topographical features are held stationary at various locations in the vicinity ano within the contact while the disk is rotating. It is shown that these topographical features can cause substantial changes in the traction when compared to traction obtained with smooth surfaces.
\end{abstract}

\section{INTRODUCTION}

Some degree of sliding occurs in essentially all concentrated contacts. With the existence of sliding, the contacts are generally subjected to conditions which are more severe than under pure rolling conditions and thus contact failures are more likely to occur. The presence of sliding in elastonydrodynamic (EHD) contacts not only results in frictional heat generation and possible thermai-associated contact failures, but it also produces tangential forces which infiluence both fatigue and skidding failures in rolling element bearings. Furthermore, the presence of sliding can increase the micro-EHD action associated with irregularities in the contacting surfaces [1-3]. This action can change locally the film thickness and pressure discribution and, therefore, traction in the contact.

For the reasons stated above and because of recent interest in traction drives, it is important to accurately predict traction in EHU contacts. Most of these predicions in the past have been obtained by considering contacts whert full-film EHD conditions existed, i.e., where surface roughness played a minor $10 l e[4,5]$. Since it is widely recognized that, in practice, most mechanical eiements do not continuously operate under such ideal conditions, there have bren recent efforts to more fully understand the role surface topograrhy plays in traction developed in EHD contacts [6-3:].

The purpose of the present work is to report on the effects of surface irregularities, in the form of dents and grooves, on the traction in siding EHD point contacts. In previous investigations [1-3] it was noted that these surface irregularities could cause large film trickness variations in the vicinity of the irregularities as well as throughout the contact. From these film thickness variations, it can be implied that traction could also change. Even through an exhaustive experimental program has not been con- 
ducted, the results to be presented give some indication as to traction changes caused by surface irregularities as these irregularities approach and pass through the contact. As in previous investigations [1-3], the contact was formed with a ball loaded against a transparent disk. The irregularities or defects are formed on the ball and held stationary at various positions in the inlet region and Hertzian region while the disk is moving. Both film thickness and traction were monitored sinultaneously.

\section{EXPERIMENTAL APPARATUS AND TrST MATERIALS}

Both traction and film thickness were measured by using the optical elastohydrodynamic apparatus described in detail in references $[9,10]$. The traction Detween the ball and the disk was obtained by measuring the reaction force on the ball. Film thickness measurements were made by means of optical interferometry. Details on the film thickness measuring system and its calibration are described in $[11,12]$. All measurements were carried out at room temperature.

The test materials, including test balls, disk and lubricant, are the same as those used in [3]. In [1], details are given on the formation of the artificial defects on the balls.

\section{PROCEDURE}

The surface defects were positioned at various locations in the conjunction region and traction data were taken for each location. At the same time, single-flash photographs were taken of the contact to aetermine the film thickness variations downstream of the defects as done in [?]. Stylus traces through the deepest part of the "undeformed" defects were also taken after the testing program on each defect had been completed. For the figures which will be presented, the inlet data ure at the right-hand side of the figure and the exit data are at the left-hand side. The defects are progressively positioned from the inlet to exit regions.

\section{RESULTS}

Stylus traces through the deepest part of all the defects studied, except the longitudinal groove, are inserted in figures showing the corresponding traction data. Three paraneters are used to specify the geometry and position of the defects. The position is specified by $\bar{x}=x / a$ where $x$ is the distance from the center of the Hertzian contact to the center (deepest part) of dents or the single transverse groove, or to the center (deepest part) of the groove closest to the contact for multiple transverse grooves and " $a$ " is the contact radius. The wioth of the defects is specified by $C=c / a$ where $c$ is one-half of the largest width of single defect or one-half of the average of the largest widths of multiple transverse grooves. The normalized depth of the defects is sperified by the parameters $\Delta=\delta / h_{0}$ where $\delta$ is the maximum depth of single defects or the average maximum depths for multiple transverse grooves and $h_{0}$ is the central film thickness based on smooth surfaces. Because most of the deferts have built-up edges, the value of $c$ and $\delta$ are obtained by extending the simooth-surface profile on both sides of the defect, as indicated in the top stylus trace insert shown in Fig. 1(a). Also note from this insert that in approximating the value of $c$, the rounded euges (left-hand side of the defect) are not considered. 
In interpreting all data to be presented, two considerations should be kept in mind. First, the lines connecting any two data points, especially for dents, do not necessarily represent actual trends between these points since mure data points might be needed to completely describe changes in both film thickness and traction. Second, in some cases, only approximate film thickness data are given. These approximations are necessary since film thickness calibration data are only avilable at descreet points which correspond to given fringe colors. 0ccasionally, the colors in the contact are in between the colors which represent known $f i l_{n i}$ thicknesses and therefore educated guesses have to be made as to the actual film thickness. In addition, it should be emphasized that the downstream film thickness for the dents represents the film thickness in a band downstream of the dents. As in [3], dents considered in this paper to not change the film thickness throughout the contact. Both traction and film thickness data are normalized on their smooth surface vílues which are represented by a zero subscript.

The inserted iraces shown in Fig. 1(a) represent dents. The top trace represents a dent which is geomeirically similar to that shown in Figs. 5 and 6 of [3] while the bottom trace represents a dent which is a little smaller than that shown in Figs. 7 and 8 of [3]. Figure 1(a) shows that the presence of surface dents generally gives a traction furce which is above that obtained for smooth surfaces. A transition from higher to lower traction is observed when the dents are located at the leading edge of the Hertzian region $(\bar{x} \simeq 1)$. The dents in this position can change the shape of the inlet region and enhance the generation of inlet pressure as implied by the $\mathrm{film}$ thickness data given in Fig. 1(b). From this figure, it is seen that with the dents located near $\bar{x}=1$, the film thickness downstream of the dents can be much larger than the film thickness based on smooth surfaces. With such large increases in downstream film thickness, the traction force can even be less than the smooth surface value as indicated in Fig. 1(a) for the largest size dent. However, with dents positioned at any other location near the inlet and inside the contact, the traction is nigher than that based on smooth surfaces even though, at some points, the downstream film thickness is larger than the corresponding snlooth surface value. Such increases are associated with local reductions in film thickness in the vicinity of the dents plus local pressures which can be much nigher than the pressure generated with smooth surfaces. Figure 1 shows that the larger dent causes more local film thickness reduction and therefore larger relative traction forces.

A stylus trace of a relatively shallow groove oriented transversely to the direction of flow is inserted in Fig. 2(a). The geometry of this groove is similar to grooves studied in [1-3]. The traction and film thickness data for this groove are given in Fig. 2. Unlike dents, for which the film thickness can locally excied the smooth surface value and traction can be below the smooth surface values, it is seen that for the groove examined in this paper, the film thickness is below or equal to the smooth surface value while the traction is always above the smooth surface value. Note that the film thickness data given for $h_{0}=0.16 \mu m$ in Fig. $2(b)$ seem to indicate that, in the range $0<\bar{x}<1$, the film thickness can be significantly lower than the smooth surface value. However, as previously stated, the line drawn in this range does not necessarily represent the actual film thickness distribution. It is felt that if a data point was available for an $\bar{x}$ of approximately 0.85 , the film thickness distribution for $h_{0}=0.16 \mathrm{\mu m}$ would be sinilar to that for $h_{0}=0.26 \mu \mathrm{m}$. 


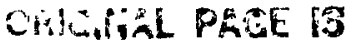 \\ OF POOR QUALITY}

Traction changes associated with a groove oriented longitudinal to the direction of flow, with $C=0.15$ and $\Delta=2.7$ (based on $h_{0}=0.26 \mu \mathrm{m}$ ), was also investigated using the same operating conditions used for the previous groove (disk velocity $=0.107 \mathrm{~m} / \mathrm{s}, P_{\max }=1.13 \times 10^{9} \mathrm{~N} / \mathrm{m}^{2}$ and $\left.T=23.3^{\circ} \mathrm{C}\right)$. When the groove traversed the, hole contact, the ratio of the traction under this condition to the traction based on a smooth surface, Fic o, was measured as 1.03. Comparing this traction value to the traction values for the previous transverse groove, it can generally be stated that under the same operating conditions, transverse grooves cause a larger increase in traction than longitudinal grooves. Such a difference can be explained by the fact that, with the logitudinal groove, the micro-EHD contribution to traction is less because the variation in surface profile is parallel to the fluid flow rather than perpendicular as it is witn the transverse grooves.

The insert shown in Fig. 3(a) shows a stylus trace of three grooves which are oriented transversely to the direction of flow. The average value of $C$ for these grooves is 0.14 while the average value of $\Delta$ is 2.6 for $h_{0}=0.16 \mu \mathrm{m}$ and 1.6 for $h_{0}=0.26 \mu \mathrm{m}$. Photographs of interference fringes of the same three grooves as they approach and go through the contact are given in Fig. 19 of [3]. The traction and downstream film thickness data, as functions of the position of the groove nearest the inlet, are given in Fig. 3. As with the single transverse groove, the film tnickness is always lower, as the grooves enter and go through the contact, than the film thickness based on smooth surfaces. The increase in traction with the three grooves, as shown in Fig. $3(a)$, is relatively large compared with the single groove described previously. The change in traction from the smooth surface value increases ds the initial central film thickness decreases. Note that the same trend is not seen in Fig. 2(a) where the smaller initial central film thickness resulted in a slightly smaller relative traction over most positions of the contact One possible explanation for these different trends is that the groove used to obtain the data shown in Fig. 2(a) caused only minor changes in the downstream film thickness once it entered the contact. Therefore, the slight ly lower relative tractions observed in Fig. $2(a)$ for $h_{0}=0.16 \mu \mathrm{m}$ might be caused by increasingly significant thermal effects, in the neighborhood of the groove, as the central film thickness is reduced. The same reasoning cannot be applied to the traction data given in Fig. 3(a) since the three grooves cause large film thickness variations from the smooth surface value irrespective of their location within the contact.

\section{DISCUSSION}

The main purpose of this paper has been to provide some data on the effects of artificially produced surface irregularities such as dents and grooves on the traction in sliding EHD point contacts.

It should be noted that the traction data presented were obtained under pure sliding conditions. The influence of the surface irregularities examined in this paper on traction could not be measured under combined rolling and sliding conditions because the transient time for these irregularities to pass through the contact is too short relative to the inertia response time of the traction measurment system. It is well known that only a small sliding component is needed for the tracation coefficient to reach its maximum value, especially for heavily loaded contacts. If the traction is derived on a micro-level in the same way as the macro-level, it is reasonable 
to assume that the relative change in traction for low slide/roll ratios will be similar to pure sliding.

It is shown that relatively small surface irregularities whose average depth is of the order of $0.4 \mu \mathrm{m}$ can increase traction, based on smooth surfaces, by 20 to 25 percent. It is interesting to note that, after making some simplifying assumptions, Tallian [ 6$]$ predicts a 25 percent increase in traction for low slide/roll ratios due to pressure ripples for a film thickness to surface roughness ratio of two. These changes are significant considering recent interests in accurately predicting traction in traction drives. In addition, such changes in traction indicate much larger localized changes in shear stresses which can have significant effects on asperity plastic flow and scuffing in concentrated contacts.

As expected, grooves oriented transversely or longitudinally to the direction of flow will inciease the traction relative to the smooth surface value under the same operating conditions. This increase, however, tends to be larger for transverse grooves than for longitudinal grooves. Such trends have also been observed in hydrodynamic lubrication [13]. With dents, it is possible to obtain traction below the smooth surface value when the dents are positioned in the inlet region. This decrease is caused by a large local filin thickness increase in the contact region as a result of a $f$ avorable modification of the inlet shape. However, this decrease of traction tends to be relatively small and it occurs only when the gents are positioned over a small portion of the contact in the inlet region. Over the rest of the contact, traction is larger than the smooth surface value.

The three grooves inserted in $\mathrm{Fig.} \mathrm{3(a)} \mathrm{have} \mathrm{an} \mathrm{average} \mathrm{depth} \mathrm{(measured}$ from the smooth surface) of approximately $0.41 \mu \mathrm{m}$. Because of their close spacings, two adjacent grooves also produce summits relative to the smooth surface. The left summit is approximately $0.1 \mu \mathrm{m}$. The average spacing of the summits is $44 \mu \mathrm{m}$ while the average slope is $1.7^{\circ}$. The surface topography produced by these three grooves is not drastically different from the fundamental roughness found in real surfaces [14]. A comparison of a ground surface taken from [15] to these grooves is shown in Fig. 4. Since the horizontal scale is about the same, note that the main structure (waviness) of the ground surface (indicated by a dashed line) is approximately the same as that of the three grooves. The ground surface is actually "rougher" $(0=0.5 \mu \mathrm{m}$ rins) than the grooved surface $(0=0.2 \mu \mathrm{m} \mathrm{rms})$. In addition, since the central film thickness (based on smooth surfaces of $0.16 \mu \mathrm{m}$ and $0.26 \mathrm{um}$ are approxinately the same as the neights of the summits, the lubrication conditions analyzed are not much different that what $c$ an be expected in practice. Therefore, the traction data presented for the three grooves should give a good indication as to the difference between traction produced by real rough surfaces having approximately the same fundamental roughness and traction based on smooth surfaces if the operating conditions are approxinately the same.

The changes in traction from the smooth surface value can be considered to be composed of two components. One of these components results from changes in the inlet surface geometry as the dents and grooves approach the inlet region. Such changes lead to lower or higher inlet pressure and film thickness depending on the position of the defects and their geometr:. These variations of pressure arid film thickenss will influerice traction. The second component results from micro-EHD pressure generation when the defects are in the Hertz region. This inicro-EHO action redistributes the pressure so that the load is mainly supported by the converging regions of 
the defects. This implies higher local pressures, temperature and shear in these regions resulting in possible asperity plastic flow, micro-pitting, chemical interictions between the surfaces and lubricant and perhaps initiation of scuffing.

\section{CONCLUSIONS}

The following observations can be made as the result of this study:

1. With dents, it is possible to have traction lower than the smooth surface value when they are positioned within a narrow range in the inlet region of the contact. When the dents are positioned throughout the rest of the contact, traction is always larger than the smooth surface value.

2. with both transverse and longitudinal grooves, the traction increases relative to the smooth surface value as these defects enter and go through the contact. Greater increases are observed with transverse grooves than with longitudinal grooves.

3. Even with irregularities having an average peak-to-peak height of approximately $0.4 \mu \mathrm{m}$, the traction $c$ an be increased by as much as 20 to 25 percent over the smooth surface value.

4. Considering the significant changes in traction causea by dents and especially grooves, it can be deduced that much larger changes in localized shear stresses occur as the result of these defects. These changes can significantly contribute to the initiation of scuffing failures in concentrated contacts.

\section{REFERENCES}

1. Wedeven, L. D., and C. Cusano, "Elastohydrodynamic Film Thickness Measurements of Artificially produced Surface Dents and Grooves," ASLE Trans., 22 (4), 369-381 (1979).

2. Cusano, C., and L. D. Wedeven, "Elastohydrodynamic Film Thickness Measurements of Artificially produced Nunsmooth Surfaces," ASLE Trans., 24 (1), 1-14 (1981).

3. Cusano, C., and L. D. Wedeven, "The Effects of Artificially produced Defects on the Film Thickness Distribution in Sliding EHD Point Contacts," paper presented at the ASLE/ASME Joint Lubrication ionf., New Orleans, La., 4-7 Oct. 1981; ASME paper No. 81-Lub-46.

4. Jefferis, J. A., and K. L. Johnson, "Sliding Friction between Lubricated Rollers," Proceedings of the Insitution of Mechanical Engineers, 182, Part 1, 281-291 (1968).

5. Johnson, K. L. and J. L. Tevaarwerk, "Shear Behavior of Elastohydrodynamic 0 il Films," Proceedings of the Royal Society of Lolidon, A356, 215-236 (1977).

6. Bair, S., and W. D. Winer, "Regimes of Traction in Concentrated Contact Lubrication," Paper presented at the ASLE/ASME Joint Lubrication Conf., New Or leans, La., 4-7 0ct. 1918; ASME Paper no. 81-Lub-10.

7. Nemlekar, P. R., and H. S. Cheng, "Traction in Rough Elastohyorodynamic Contact," Symposium sponsored by the Lubrication Division of ASME, Chicago, 111.. 16-21 Nov. 1980.

8. Tallian, T. E., "Pressure and Traction Rippling in Elastohydrodynamic Contact of Rough Surfaces," Journal of Lubrication Technology, ASME Trans., 96, Series $F(3), 398-409$ (1974). 
9. Wedeven, L. D., "Traction and Film Thickness Measurements under Starved Elastohydrodynamic Conditions," Journal of Lubrication Technology, ASME Trans., 97, Series F (2), 321-329 (1975).

10. Wedeven, L. D., "Effect of Starvcation on Film Thickness and Traction under Elastohydrodynamic Rolling and Sliding Conditions," NASA TN D-8087 (1975).

11. Wedeven, L. D., D. Evans, and A. Cameron, "Optical Analysis of Ball Bearing Starvation," Journal of Lubrication Technology, ASME Trans., 93, Series $F(3), 349-363(1971)$.

12. Foord, C. A., L. D. Wedeven, F. J. Westlake, and A. Cameron, "uptical Elastohydrodynamics," Proceedings of the Institute of Mechanical Engineers, 184, Part 1, (28), 487-505 (1969-1970).

13. Hata, H., T. Nakahara, and H. Aoki, "Measurement of Friction in Lighly Loaded Hydrodynamic Siiders with Striated Roughness," Symposium sponsored by the Lubrication Division of ASME, Chicago, I11., 16-21 Nov. 1980.

14. Tallian, T. E., "The Theory of Partial Elastohydrodynamic Contacts," Wear, 21, 49-101 (1972).

15. Whitehouse, D. J., and J. F. Árchard, "The Properties of Ranoom Surfaces of Significance in the ir Contact," Proc. Roy. Soc. of London, A.316, 97-121 (1970). 


\section{ORIGINAL PAGE IS \\ OF POOR QUALITY}

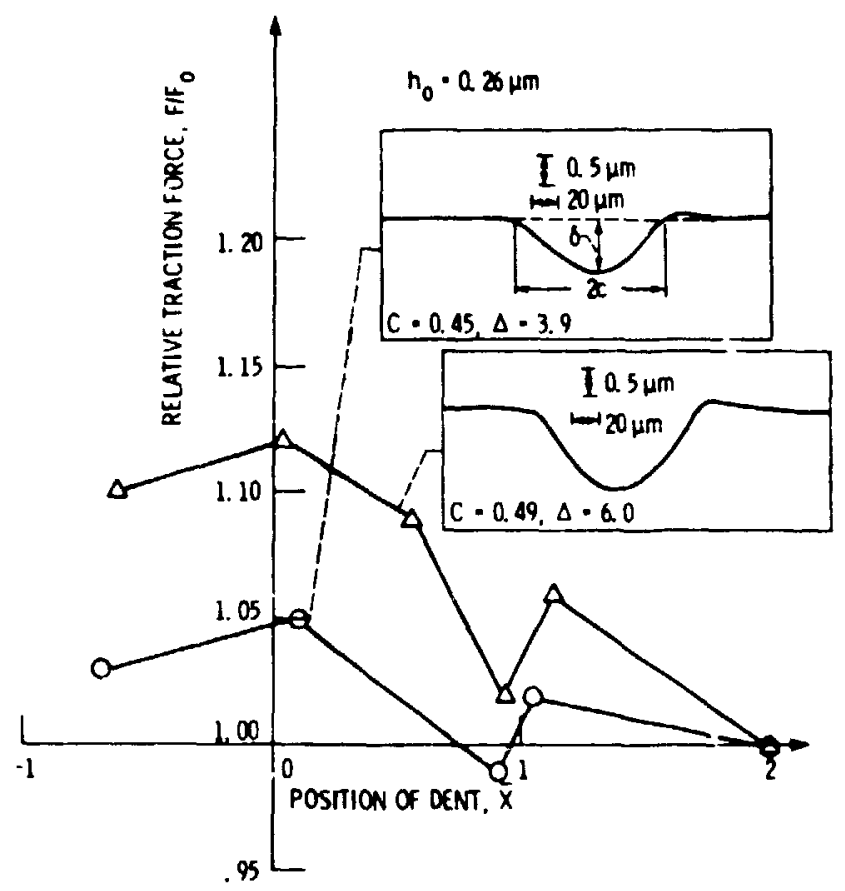

(a) Relative traction as a function of dents cosition.

Figure 1 . Relative traction and flim thickness as a function of dents position. Disk velocity $-0.107 \mathrm{~m} / \mathrm{s} . P_{\max }=1.13 \times 10^{9} \mathrm{~N} \mathrm{~m}^{2} .1 \cdot 23.3^{\circ} \mathrm{C}$.

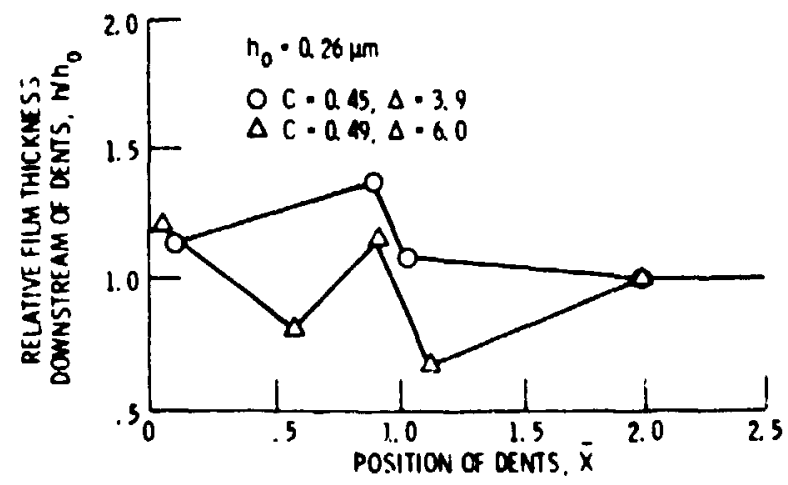

(b) Relative film thickmess as a function if conts position.

Figure 1. - Conciuded. 


\section{ORIGINAL PAẼ: IS
OF POOR QUALITY}

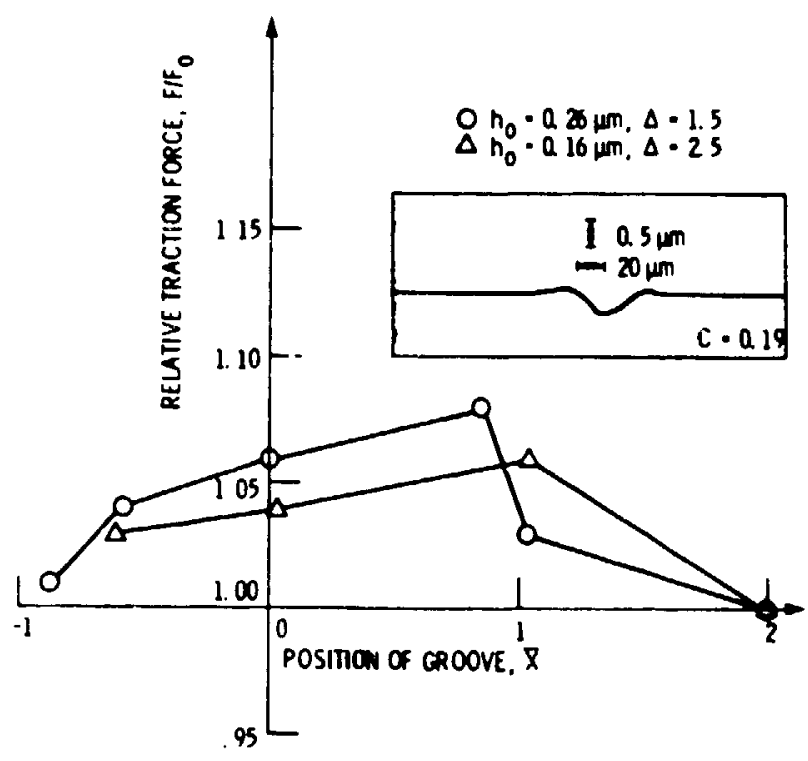

(a) Relative traction as a function of groove position.

Figure 2 - Relative traction and film thickness as a function a groove Dosition Disk velocity $\cdot 0.100 \mathrm{w} / \mathrm{s}$ for $n_{0} \cdot 0.26 \mathrm{um}$ and $Q .037 \mathrm{~m} / \mathrm{s}$ for $n_{0} \cdot 0.16 \mathrm{~mm}, P_{\max } \cdot 1.13 \times 10^{\circ} \mathrm{Nm}^{2}, \mathrm{~T} \cdot 22.3^{\circ} \mathrm{C}$.

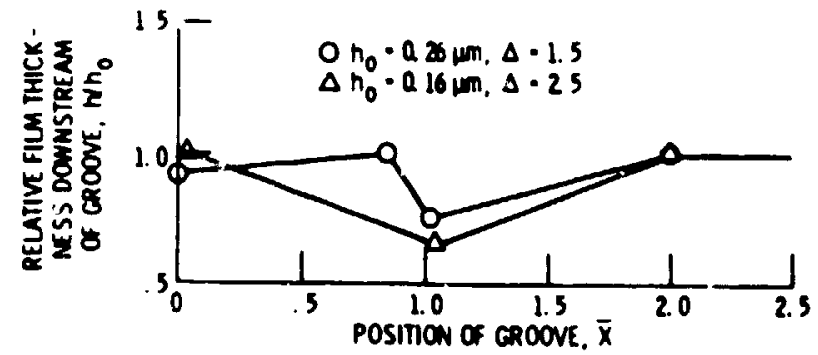

(b) Reiati ie film thickness as a function a groove position.

Figure 2 - Concluad 


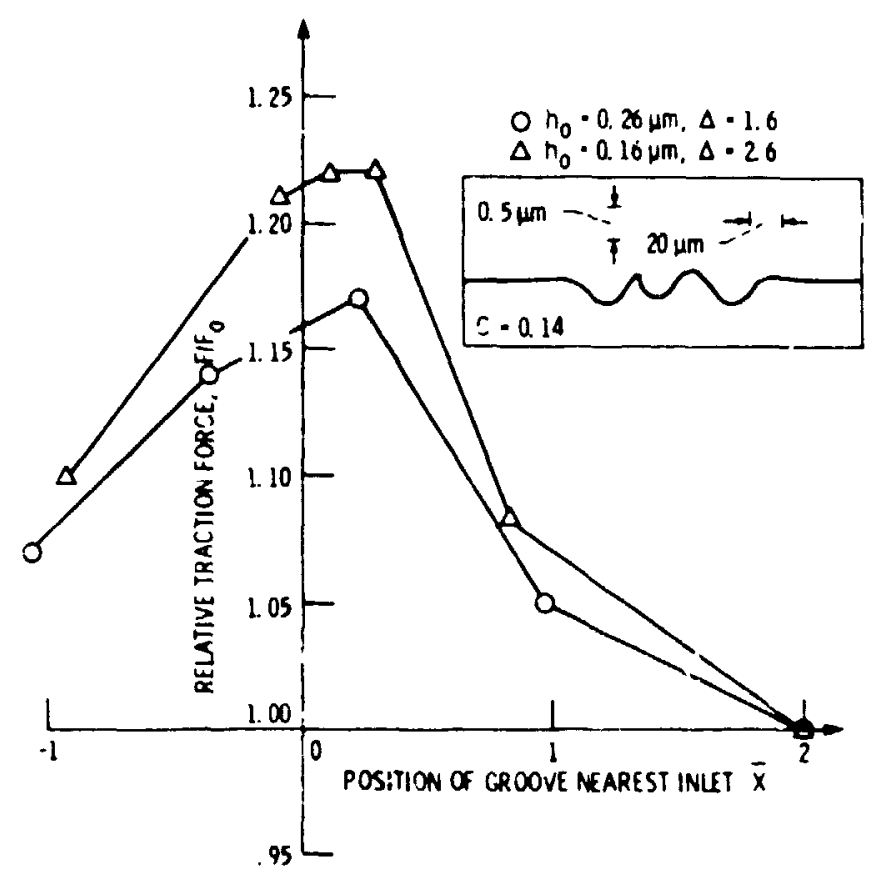

(a) Relative traction as a function of grooves position.

Figure 3. - Relative traction and film inickness as a function a grooves position. Disk velocity $\cdot 0.10 \mathrm{~m} / \mathrm{s}$ for $h_{0} \cdot 0.26 \mathrm{~mm}$ and $0.037 \mathrm{~m} / \mathrm{s}$ for $h_{0}=0.16 \mathrm{~mm}, P_{m=x}=1.13 \times 10^{9} \mathrm{~N} / \mathrm{m}^{2}, \mathrm{r}=23.3^{\circ}, \mathrm{C}$.

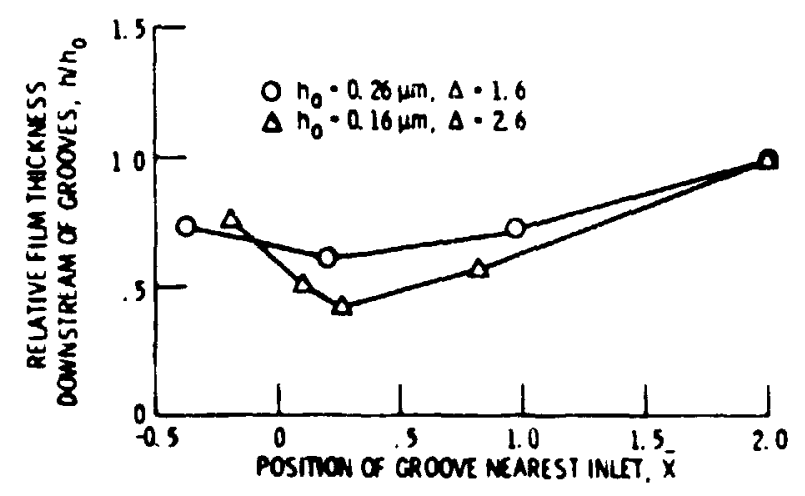

(b) Relative lilini Eickness as a lunction al growes position.

Figure 3. - Concludere. 
CRICINAL PAGE IS

OF POOR QUAL!TY
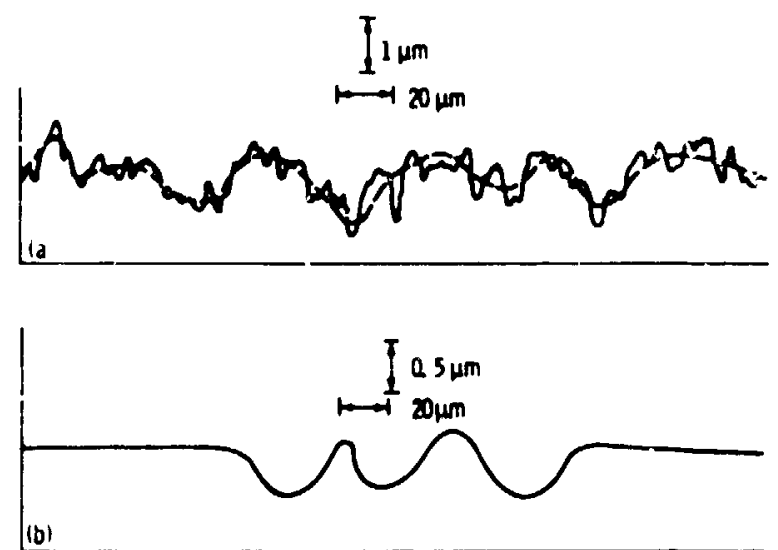

(a) Stylus trace of a typical ground surtaca $\mid 15)$.

(b) Three growes used to obtain ath given in flg 3.

Figure 4 - Comparison a a halcal ground surtece with artiliclally produced surfece topography. 